\title{
Clinical utility of custom-designed NGS panel testing in pediatric tumors
}

\author{
Lea F. Surrey ${ }^{1,2 \dagger}$, Suzanne P. MacFarland ${ }^{3 \dagger}$, Fengqi Chang ${ }^{2}$, Kajia Cao ${ }^{2}$, Komal S. Rathi ${ }^{4,5,6}$, Gozde T. Akgumus ${ }^{2}$, \\ Daniel Gallo2, Fumin Lin², Adam Gleason², Pichai Raman ${ }^{4,5,6}$, Richard Aplenc ${ }^{3,5,7}$, Rochelle Bagatell ${ }^{3,5,7}$, \\ Jane Minturn 3,5,7, Yael Mosse ${ }^{3,5,7}$, Mariarita Santi ${ }^{1}$, Sarah K. Tasiann 3,5,7, Angela J. Waanders 3,4,5,7, Mahdi Sarmady, ${ }^{1,2}$, \\ John M. Maris ${ }^{3,5,7}$, Stephen P. Hunger ${ }^{3,5,7}$ and Marilyn M. Li ${ }^{1,2,7,8^{*}}$ (D)
}

\begin{abstract}
Background: Somatic genetic testing is rapidly becoming the standard of care in many adult and pediatric cancers. Previously, the standard approach was single-gene or focused multigene testing, but many centers have moved towards broad-based next-generation sequencing (NGS) panels. Here, we report the laboratory validation and clinical utility of a large cohort of clinical NGS somatic sequencing results in diagnosis, prognosis, and treatment of a wide range of pediatric cancers.

Methods: Subjects were accrued retrospectively at a single pediatric quaternary-care hospital. Sequence analyses were performed on 367 pediatric cancer samples using custom-designed NGS panels over a 15-month period. Cases were profiled for mutations, copy number variations, and fusions identified through sequencing, and their clinical impact on diagnosis, prognosis, and therapy was assessed.

Results: NGS panel testing was incorporated meaningfully into clinical care in $88.7 \%$ of leukemia/lymphomas, 90. $6 \%$ of central nervous system (CNS) tumors, and $62.6 \%$ of non-CNS solid tumors included in this cohort. A change in diagnosis as a result of testing occurred in 3.3\% of cases. Additionally, 19.4\% of all patients had variants requiring further evaluation for potential germline alteration.
\end{abstract}

Conclusions: Use of somatic NGS panel testing resulted in a significant impact on clinical care, including diagnosis, prognosis, and treatment planning in $78.7 \%$ of pediatric patients tested in our institution. Somatic NGS tumor testing should be implemented as part of the routine diagnostic workup of newly diagnosed and relapsed pediatric cancer patients.

Keywords: Tumor sequencing, Molecular profiling, Pediatric cancer

\section{Background}

Cancer is the second leading cause of death in children, and pediatric cancers are a diverse set of malignancies with pathologic and clinical heterogeneity based on age of onset and underlying tumor biology. Some pediatric cancers are nearly exclusive to the childhood age group, such as neuroblastoma, Wilms tumor, and atypical teratoid rhabdoid tumor. Others are diagnosed in both adults and children

\footnotetext{
* Correspondence: lim5@email.chop.edu

${ }^{\dagger}$ Lea F. Surrey and Suzanne P. MacFarland contributed equally to this work ${ }^{1}$ Department of Pathology and Laboratory Medicine, The Perelman School of Medicine at the University of Pennsylvania, Philadelphia, PA 19104, USA

2Division of Genomic Diagnostics, Children's Hospital of Philadelphia, Philadelphia, PA 19104, USA

Full list of author information is available at the end of the article
}

but have unique features and genomic profiles specific to pediatric populations, such as B-acute lymphoblastic leukemia (ALL) and diffuse astrocytoma. Several large sequencing studies have broadly characterized the genomic landscape of childhood cancers, and individual reports have expanded tumor-specific genetic patterns $[1,2]$. Pediatric tumors have a relatively low mutational burden at diagnosis and higher rate of driver gene fusions when compared to adult tumors, while in adult tumors the somatic mutation rate ranges from 1 to $10 / \mathrm{Mb}$, pediatric tumor average mutation rates range from $0.17-0.79 / \mathrm{Mb}$ [2-5]. Recent studies have identified new tumor-specific and non-specific driver genes, gene signatures for specific tumor types, and a defined

(c) The Author(s). 2019 Open Access This article is distributed under the terms of the Creative Commons Attribution 4.0 International License (http://creativecommons.org/licenses/by/4.0/), which permits unrestricted use, distribution, and reproduction in any medium, provided you give appropriate credit to the original author(s) and the source, provide a link to the Creative Commons license, and indicate if changes were made. The Creative Commons Public Domain Dedication waiver (http://creativecommons.org/publicdomain/zero/1.0/) applies to the data made available in this article, unless otherwise stated. 
mutational spectrum in a wide variety of pediatric cancers $[1,2]$.

Somatic genomic testing has become the standard of care in a variety of pediatric cancers for diagnostic refinement, risk stratification, and therapeutic approach, such as the incorporation of $M C Y N$ amplification, DNA ploidy, and segmental chromosomal aberrations in International Neuroblastoma Risk Group classification of neuroblastoma and the use of genetic profiling in World Health Organization (WHO) classification of central nervous system (CNS) malignancy [6-10]. Identification of somatic mutations, fusions, and other genomic aberrations has led to implementation of molecularly targeted therapies in several pediatric cancers, including Philadelphia chromosome positive $(\mathrm{Ph}(+))$ and $\mathrm{Ph}$-like acute lymphoblastic leukemia and ALK-mutated neuroblastoma [11, 12]. Clinical trials have begun to incorporate genomic profiling into selection of targeted agents [13].

While large whole-exome and whole-genome sequencing studies have given us new insights into pediatric cancers as a whole, few of these approaches are offered by clinical laboratories to guide routine clinical practice. Large, low-cost, and short turnaround time (TAT)-targeted cancer panels have become widely available from clinical laboratories, including some that are FDA approved or cleared $[14,15]$. However, these have typically been developed to detect the spectrum of mutations present in adult cancer, and often, genes important in pediatric cancer are not interrogated. Our laboratory has designed, validated, and implemented comprehensive-targeted sequencing panels that cover single nucleotide variants (SNV), small insertions/deletions (indel), copy number alterations (CNV), and fusion genes that are recurrent in pediatric (and often adult) cancers. Despite the availability of large targeted cancer panels at our institution and elsewhere, the clinical utility of comprehensive somatic sequencing panels is still relatively limited in the pediatric cancer population [16-24]. Other studies have evaluated the use of whole-exome/transcriptome sequencing in the pediatric oncology population to identify clinically actionable variants in both the upfront and relapsed settings $[17,19,21]$, as well as the feasibility of real-time molecular diagnosis from tumor specimens [22]. We report the performance of these NGS-based somatic panels as a part of clinical care of a broad variety of newly diagnosed and relapsed pediatric cancer patients and assess the analytical validity and clinical utility for pediatric tumor diagnosis, prognosis, and therapeutic decision-making.

\section{Methods}

\section{NGS-based targeted DNA and RNA sequencing} Panel design

Ninety-nine genes associated with pediatric hematological malignancies, 237 genes associated with pediatric solid tumors, and 106 major fusion partner genes associated with cancer-related fusions are selected for the CHOP Comprehensive Hematological Malignancy Panel (CHMP) and Comprehensive Solid Tumor Panel (CSTP). Custom RNA probes were designed using SureDesign (Agilent Technologies, Santa Clara, CA) to cover all exons and at least $10 \mathrm{bp} 5^{\prime}$ and $3^{\prime}$ flanking intronic sequences, and selected known intronic mutations (Additional file 1: Table S1). The panels also contain probes covering at least a 300-bp TERT promoter region [25] as well as four single nucleotide polymorphisms (SNPs) in TPMT (rs1142345, rs1800462 and rs1800460) and NUDT15 (rs116855232) genes associated with increased risk of thiopurine-related toxicities [26, 27]. An additional 1038 common SNPs were added to both CHMP and CSTP to mimic a low-density SNP array for CNV analysis [10]. All custom RNA probes were synthesized and biotinylated to allow for target enrichment using streptavidin-conjugated beads (Agilent Technologies). For targeted RNA sequencing, custom DNA primers were designed using ArcherDX Assay Designer (ArcherDX, Boulder, CO) to cover 586 known fusion transcripts and potential novel fusions associated with 106 cancer genes.

\section{Nucleotide extraction}

Nucleotide extraction was performed using different commercial extraction kits based on sample types and tests. The methods for nucleotide extraction are summarized in Additional file 1: Table S2. Nucleotide extractions were achieved according to the manufactures' instructions with minor modifications. For FFPE specimens, macro-dissection was used when necessary to enrich tumor content.

\section{Library construction and sequencing}

Targeted DNA sequencing libraries were constructed using SureSelect ${ }^{\mathrm{QXT}}$ Reagent Kit (Agilent Technologies, Santa Clara, CA) with $50 \mathrm{ng}$ of genomic DNA. Briefly, tumor DNA was enzymatically fragmented and tagged to generate adapter-tagged libraries. Biotin-labeled probes specific to the targeted regions of interest (ROI) via hybridization, and libraries were enriched for ROI using streptavidin beads, then amplified, dual-indexed, and pooled for sequencing; quality of the libraries were measured with 2200 TapeStation (Agilent) and quantified using Qubit 2.0 (ThermoFisher Scientific, Waltham, MA). Targeted RNA sequencing (Fusion Panel) libraries were prepared using Archer ${ }^{\mathrm{Tm}}$ Universal RNA Reagent Kit v2 for Illumina with $150 \mathrm{ng}$ of input RNA or total nucleic acid (TNA) (ArcherDX, Boulder, CO). Target-enriched library was generated by using a combination of gene-specific primers and universal adapters with minor modification to the manufacturer's instruction (ArcherDX). Briefly, RNA was reverse-transcribed to generate cDNA and 
molecular barcode adapters were ligated to cDNA followed by two rounds of target-specific PCR. The library was quantified using KAPA Biosystems qPCR kit (KAPA Biosystems, Wilmington, MA). All libraries were sequenced on Illumina MiSeq or HiSeq platform with MiSeq v2 or HiSeq Rapid SBS v2 300 cycle reagent kit (Illumina, San Diego, CA).

\section{Sequence data processing}

DNA Sequencing data were processed using Concord v1.0 (in-house developed). Briefly, paired FastQ files of each sample were aligned to the GRCh37/hg19 reference genome using Novoalign v3 (http://www.novocraft.com). PCR duplicates were marked using Picard v2.18, SAMtools [28], v1.9 converted SAM file to BAM files. Four different variant calling (SNV and Indel) tools were used: Freebayes [29] v1.0.1, VarScan2 [30] v2.3, MuTect [31] v1.1, and Scalpel (http://scalpel.sourceforge.net). For annotation and filtration, a combination of SnpEff [32] v4.3, SnpSift [33] v4.3, and in-house algorithms were used. Raw variants went through QC filtration based on strand ratios, allele fractions, mapping quality, and frequency using an internal cohort of normal controls. QC filtered variants were then merged, annotated, and filtered using various databases including COSMIC (http://cancer. sanger.ac.uk/cosmic), ClinVar (http://www.ncbi.nlm.nih. gov/clinvar/), HGMD [34], ExAC (http://exac.broadinstitute.org), and dbSNP [35]. The remaining variants were manually reviewed and classified according to the AMP/ ASCO/CAP Standards and Guidelines for Somatic Variant Interpretation and Reporting [36]. CNVs and loss of heterozygosity (LOH) analysis were performed using the CNV tool built in NextGENe v2 NGS Analysis Software (Softgenetics, State College, PA) based on normalized read counts and distribution patterns of SNPs using the SNP backbone and SNPs pulled from the ROIs. Targeted RNA sequencing data were analyzed using Archer analysis software (ArcherDX) and visualized using the JBrowse genome browser [37]. Turnaround time for this testing is approximately 3 weeks.

\section{Analytical validation}

\section{Sensitivity, specificity, and reproducibility analysis}

Analytic sensitivity, specificity, and reproducibility were evaluated by comparing SNPs and indels in the ROIs from both intra-run and inter-run of the same HapMap sample NA12878 (https://ftp-trace.ncbi.nlm.nih.gov/gia b/ftp/release/NA12878_HG001/latest/GRCh37/; Coriell Institute, Camden, NJ). Briefly, two libraries of NA12878 were prepared in parallel, each with a unique index. The libraries were pooled and sequenced in the same run. Another library of NA12878 was prepared and sequenced in a different run at a different time. The concordance of detected SNVs and indels were compared with known HapMap SNVs and indels to calculate sensitivity, specificity, and reproducibility. All known NA12878 variants in the ROI were assigned as either a true positive (TP) if detected by the assay or false negative (FN); sensitivity, specificity, and positive predictive value were calculated. The concordances of variants called between intra-runs and inter-runs for the same sample were used to calculate reproducibility. Multiple additional samples were tested, including 22 samples sequenced elsewhere, 11 samples with CNVs determined by SNP array, and 60 samples with or without fusions determined by other technologies.

\section{Determination of detection limit}

Detection limits were determined using purified DNA from five cancer cell lines (LAN6, NGP, NB-16, NB-1691 and SK-N-BE [2] C; Cell line stocks were obtained from the Children's Oncology Group (COG) Cell Culture and Xenograft Repository at Texas Tech University Health Sciences Center (www.COGcell.org), the American Type Culture Collection (Manassas, VA), or the Children's Hospital of Philadelphia (CHOP) cell line bank), 1 HapMap cell line (NA12878), and three normal control samples. Each cell line or normal control and the mixture of the cell line/normal control were sequenced and analyzed using the NGS-based cancer genome profiling workflow and analysis pipeline (Additional file 2: Figure S1). The performance of each assay was evaluated by the variant allele frequency (VAF) of the known variants in un-diluted and diluted cell lines. The CNV detection performance was evaluated using one cancer cell line DNA (LAN6) diluted with one normal control DNA and a tumor DNA sample diluted with DNA from paired normal tissue to generate pools of $50 \%, 30 \%$, and $20 \%$ tumor fractions. Each diluted sample was sequenced twice to evaluate the reproducibility.

\section{Patient characteristics}

All patient data was abstracted from patient medical records under an institutional retrospective IRB protocol. Initial data review included all patients who underwent somatic tumor testing from the period of January 2016April 2017 (15 months); all testing was ordered and conducted as a part of the clinical care of children with newly diagnosed and relapsed malignancies. A total of 389 panels on 367 different patients were initially reviewed by a team of oncologists, pathologists, and genetic counselors. Cases were removed if they were outside of the pediatric age range treated at our institution ( $0-26$ years of age) and if their final diagnosis was not oncologic, including benign hematologic disorders and specimens obtained from patients in remission without evidence of disease. 
Pathologic information was reviewed to determine oncologic diagnosis and cases were reviewed to determine the clinical relevance of the reported result to (1) support/alter diagnosis, (2) affect prognosis, and (3) potentially change therapy (use of targeted drug therapy or intensification/de-escalation based on prognosis, see below). Each case was reviewed by at least two clinicians (a pathologist and an oncologist) to determine accuracy of information obtained and to maintain consistency in reporting. Cases were categorized into leukemia/lymphoma, CNS, and non-CNS solid tumors and were further subclassified into specific tumor types.

\section{Clinical impact determination}

Clinical impact was determined based on effect on diagnosis, prognosis, and potential treatment decision-making according to currently available evidence including professional guidelines outlined by the Children's Oncology Group (COG) and the National Comprehensive Cancer Network (NCCN) among others: (1) variants qualify the patients for a FDA-approved therapy or to be enrolled in a clinical trial; (2) variants define or contribute to the diagnosis of a tumor or a subtype of tumor; (3) variants that define or contribute to patient risk stratification. Although some of the information gained from panels could also be obtained through other means (targeted sequencing, FISH), the panels provided comprehensive molecular analysis with increased efficiency avoiding the need for staged molecular testing. Results were considered diagnostically significant if they affected and/or contributed to clinical diagnosis. For CNS tumors, this would include any molecular results that contributed to the WHO integrated diagnostic criteria last updated in 2016 [8]; additionally, it provided classification data in all cases of medulloblastoma. For neuroblastic tumors, this included contributors to high-risk criteria including $M Y C N$ amplification and segmental chromosomal aberrations; for Ewings sarcoma, it included all cases with an EWS-fusion. Additionally, results that changed prognosis (e.g., changed risk classification) were also considered significant in treatment decision-making if they necessitated a change in therapy. Further, results were considered clinically significant if they provided targeted therapeutic options in the case of relapse. All cases were individually reviewed by at least one oncologist and one pathologist (with an additional oncologist and pathologist review where question arose). Somatic findings were considered potentially germline if they met several specific criteria: (1) pathogenic/likely pathogenic variants in genes known to be associated with cancer predisposition with variant allele frequency (VAF) between 40 and 100\%; (2) large indels or exonic deletions/ duplications in genes known to be associated with cancer predisposition regardless of VAF; and/or (3) suspected germline pathogenic variants regardless of VAF if the variant is a known founder mutation or if clinical features (such as tumor type) suggest that germline predisposition related to the involved variant is more likely.

\section{Data analysis}

All genomic and annotation data was processed using $\mathrm{R}$ statistical language. Specifically, the tidyverse suite of packages was used extensively to standardize, format, and summarize the data. Additionally, the biomaRt and scales packages were employed to add genomic annotations and transform the data, respectively. Most figures were generated using the ggplot 2 package with the exception of the oncoprint graphs which was created using the complexheatmap package and the fusion circos plots which were generated using OmicCircos package. Chi-square analysis was used for $p$ values. In the CNS and non-CNS solid cases, TPMT and NUDT15 pharmacogenomic variants along with MPL p.Lys29Asn (MPL Baltimore) were filtered out pre-analysis. TPMT and NUDT15 significant variants were included in analysis of therapeutic impact in liquid tumor cases. All code for analyzing and visualizing the data is available in a github repository (https://github. com/chopdgd/CHOP_CancerPanel_Analysis).

\section{Results \\ NGS panel performance}

The average sequence depths for the Comprehensive Hematological Malignancy Panel (CHMP) and Comprehensive Solid Tumor Panel (CSTP) were 1600x and 1800, respectively, for the validation samples (Additional file 2: Figure S2). The coverage of regions of interest (ROI) for both panels was at or above $99.95 \%$ at $100 x$. The sensitivity and specificity for both panels were $>99.99 \%$ for SNVs and indels with average positive predictive values of 81.23\% and $88.79 \%$ for CHMP and CSTP, respectively, for the HapMap sample (Additional file 1: Table S3). The sensitivity and specificity of the clinical samples for the reported variants were $>99.99 \%$ including 28 unique SNV/ indel events and $62 \mathrm{CNVs}$ (Additional file 1: Table S4). The detection limit was set at 5\% VAF for SNVs and indels in the Concord pipeline, and all variants at or above $5 \%$ were correctly identified. Furthermore, dilution studies showed that known variants with VAF as low as $<1 \%$ can be detected (Additional file 1: Table S5). CNV detection limit was at $30 \%$ for deletions and duplications and at least $20 \%$ for amplifications (Additional file 2: Figure S3). Reproducibility studies on cell lines, fresh, and FFPE samples showed 100\% concordance (Additional file 1: Table S6). The RNA-based fusion panel validation was performed on 60 samples in a double-blinded fashion. The panel detected all fusions identified by other methods, mostly by whole-transcriptome sequencing, and no fusions in normal controls (data not shown here). 


\section{Patient and tumor characteristics}

In total, 389 panels were performed on 367 different patients with an average age of 8.6 years (range 0-26). Diagnostic primary tumors $(n=285)$ were most commonly tested, but relapse samples are also included in this case-series $(n=104)$. Twenty patients had repeat testing of their tumor at different time points, and one patient had testing of two separate primary tumors. Overall, 2254 clinically significant Tier 1 or 2 variants of any type were detected across all panels, including 397 SNVs/indels in 101 different genes (Additional file 2: Figure S4), $1751 \mathrm{CNVs}$ (Fig. 1), and 106 fusions (Fig. 2). Only 47 panels (12.1\%) had no Tier 1 or 2 variants detected.

\section{Genomic findings and variant classification} Non-CNS solid tumors

There were 154 panels performed on 147 different patients with non-CNS solid tumors. A total of 2210 variants (SNV/indels, CNVs, fusions) were classified into Tiers 1-4 according to the AMP/ASCO/CAP Standards and Guidelines for Somatic Variant Interpretation and Reporting [36], with 48.6\% (1074/2215) as Tier 1 (variants of strong clinical significance) and Tier 2 (variants of potential clinical significance). Overall, at least one Tier 1 or 2 variant was identified in $86.3 \%$ (133/154) of solid tumors tested. Of those Tier 1 or 2 variants, CNVs were the most common alteration identified in $63.6 \%$ (98/154). Among

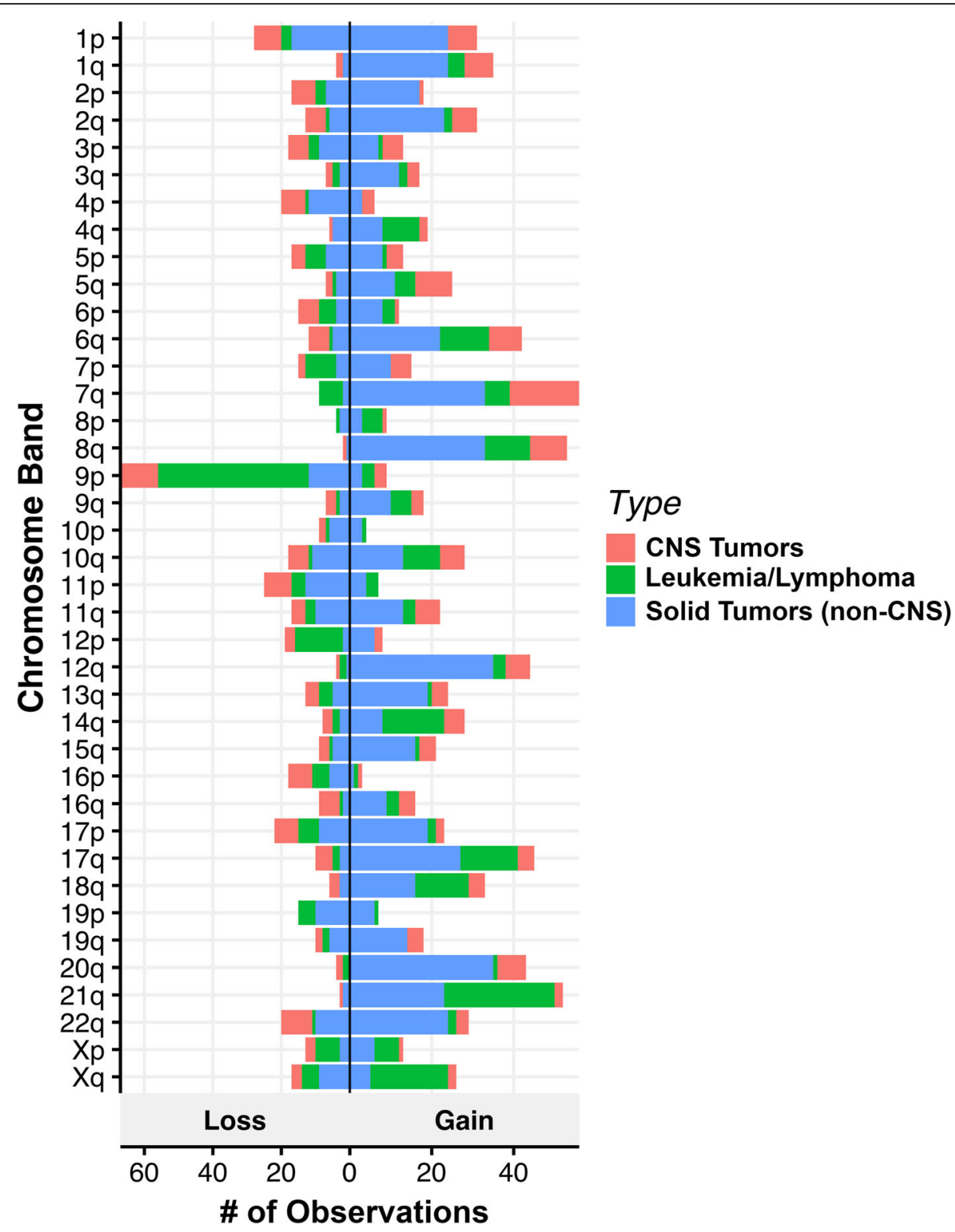

Fig. 1 Copy number alterations observed in 389 pediatric tumors. The number of observed instances of copy number gains and losses is represented on the $X$ axis vs. the chromosomal band on the $Y$ axis. Tumor types are color-coded. CNS = central nervous system 


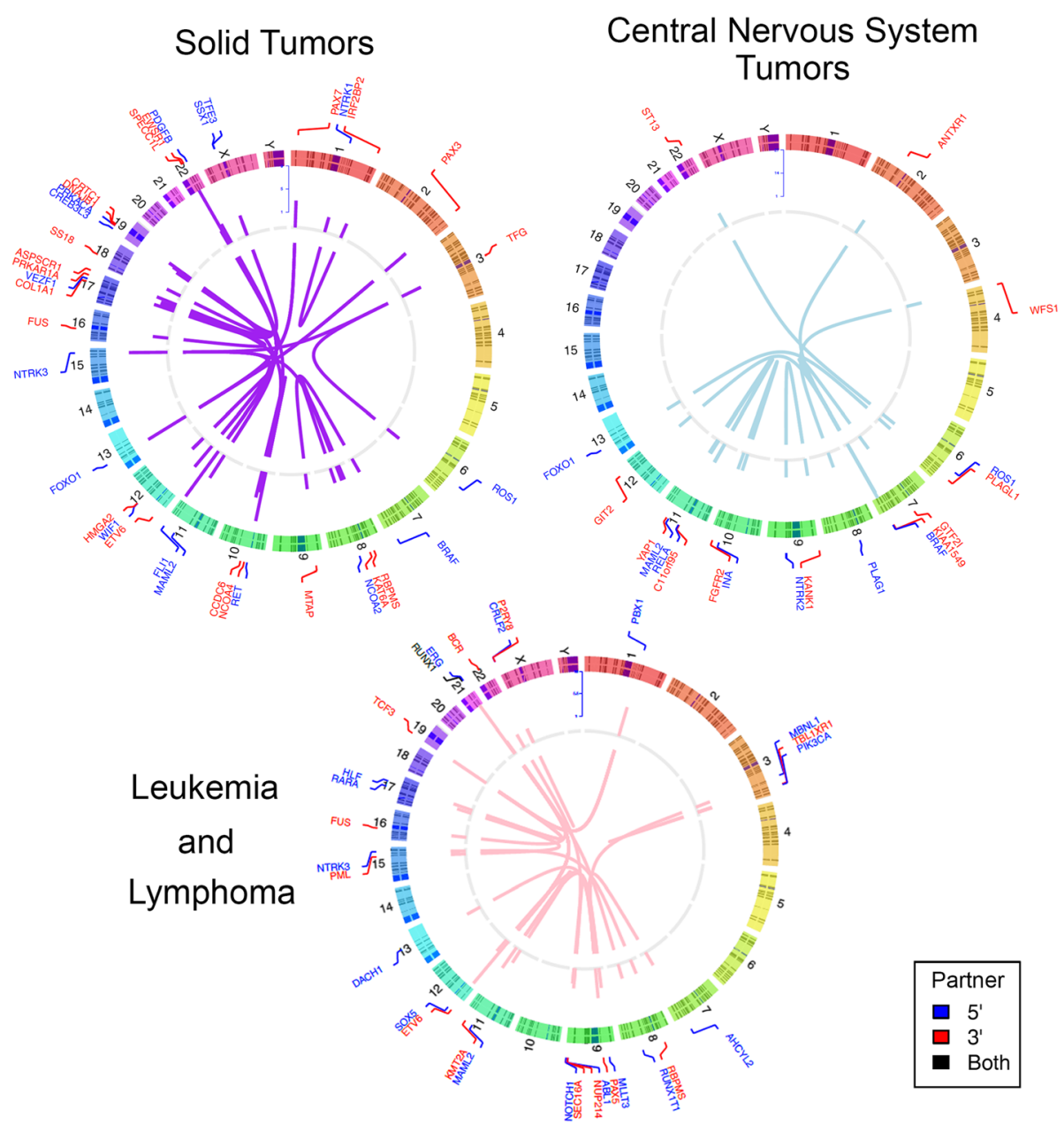

Fig. 2 Fusions detected in 389 pediatric tumors. Circos plots showing 5' (blue) and 3' (red) fusion genes across three categories of tumors tested. Genes are listed adjacent to chromosomal number and location. Central lines connect fusion partners. Details of novel/rare fusions identified can be found in Additional file 1: Table S7. CNS = central nervous system

solid tumors, gains and losses were distributed throughout the genome with variability based on tumor type (Fig. 1). Tier 1 or 2 SNVs/indels were present in 40.2\% (62/154) and fusions were present in 25.3\% (39/154). An overall summary of Tier 1 and 2 sequence and fusion variants identified in $>1$ solid tumor sample by frequency, age, and tumor type is displayed in (Fig. 3). The most common Tier 1 or 2 sequence variant was TP53, followed by $K R A S$, then $A L K$ and BRCA2 in equal numbers. Most cases contained only one Tier 1 or 2 variant, with eight tumors containing two or more clinically significant sequence alterations. Fusions were most often associated with sarcoma or carcinoma diagnosis, consisting of classic/canonical fusions as well as eight novel/rare fusions (Fig. 2, Additional file 1: Table S7).

\section{CNS tumors}

There were 101 panels performed on 96 different patients with CNS tumors. A total of 1221 variants (SNV/ indels, CNVs, fusions) were classified into Tiers 1-4, with $43.4 \%(529 / 1221)$ as Tier 1 and 2 variants. Overall, at least one Tier 1 or 2 variant of any type was identified in $92.1 \%(93 / 101)$ of all CNS tumors tested. Similar to solid tumors, CNVs were the most common alteration identified in 67.3\% (68/101) of panels. Among CNS tumors, gains and losses were distributed throughout the genome with some chromosome arms having a higher rate of either gain $(7 \mathrm{q}, 8 \mathrm{q}, 12 \mathrm{q})$ or loss $(9 \mathrm{p}$, specifically CDKN1A and CDKN2B) (Fig. 1). Tier 1 or 2 SNVs/ indels were present in $51.5 \%(52 / 101)$, and fusions were present in $33.7 \%(34 / 101)$. Low-grade astrocytomas, including pilocytic astrocytoma, were the most commonly tested tumor type, and as a result, the most common alteration was a BRAF fusion or variant (V600E or T599dup), present in 30.7\% ( $n=31 / 101$ ) of cases (Fig. 4). After $B R A F$ alterations, the most common Tier 1 or 2 sequence variants were TP53 and H3F3A K27 M. Most cases contained only one Tier 1 or $2 \mathrm{SNV} /$ indel or 


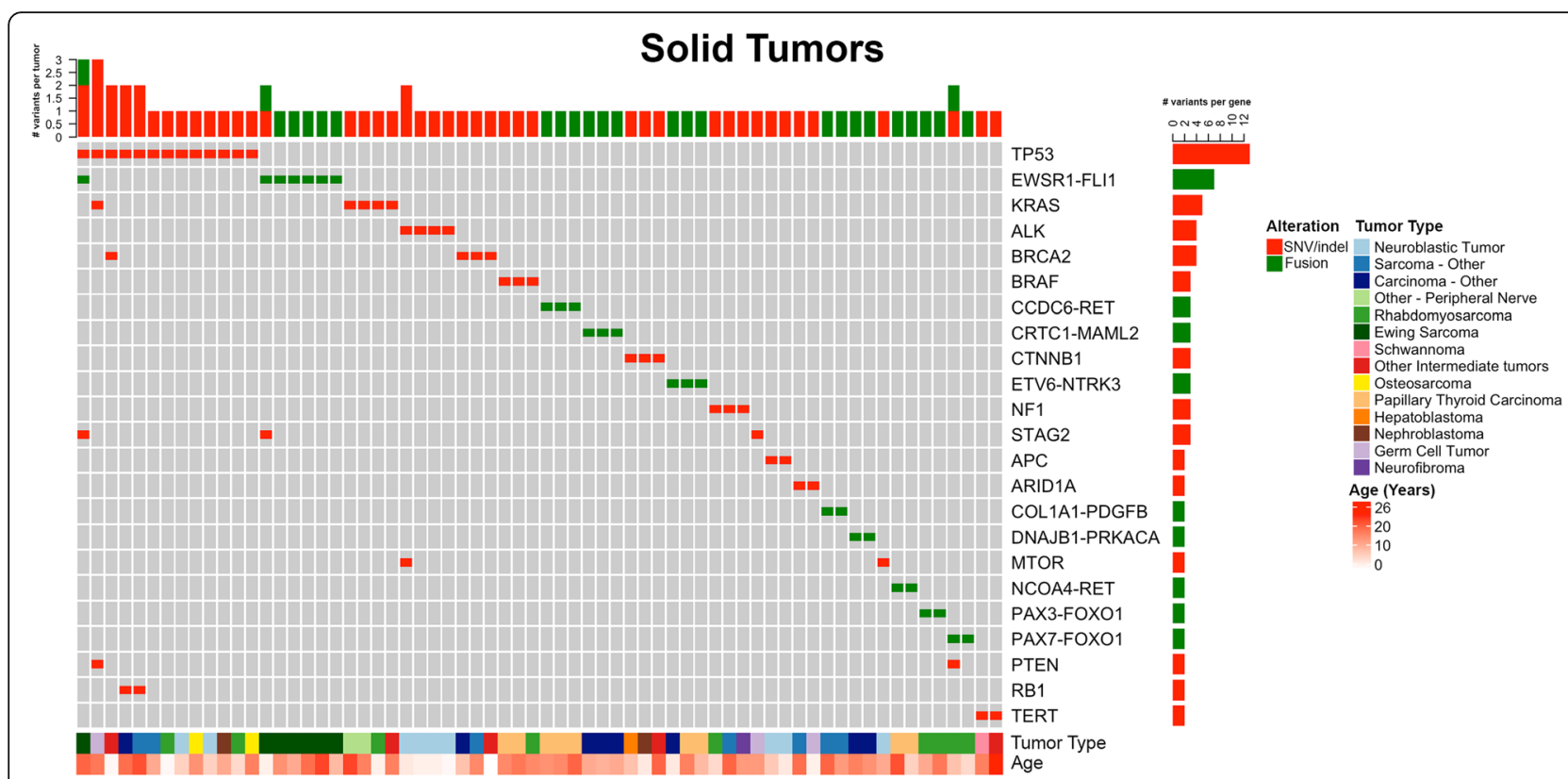

Fig. 3 Solid tumor (non-CNS) oncoprint of most common Tier 1 and 2 SNVs, idels, and fusions. Summary of most commonly encountered Tier 1 or 2 SNVs and fusion variants identified in > 1 solid tumor. TP53 was the most commonly encountered SNV, followed by KRAS, ALK, and BRCA2. EWSR1-FL11 was the most common fusion gene, followed by FOXO1 fusions, present in a total of four tumors. Overall number of clinically significant variants per tumor is represented across the top, with eight tumors have $>1$ alteration. The number of variants identified per gene is represented to the right. Age and tumor type are represented across the bottom. CNS = central nervous system, SNV = single nucleotide variant, indel = insertion/deletion

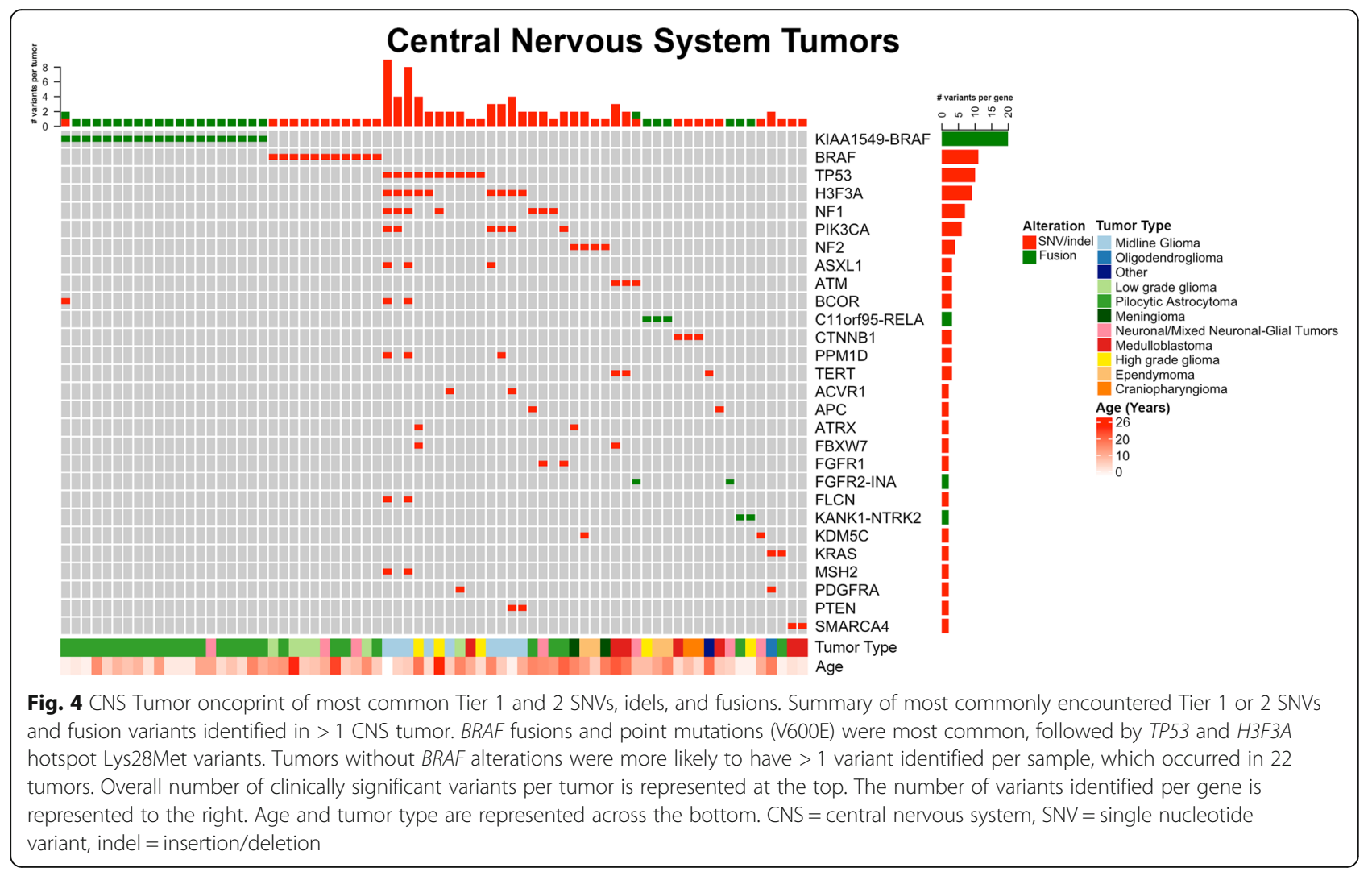


fusion, with $8 \% \quad(n=8)$ tumors containing $\geq 3$ sequence alterations (Fig. 4). Additional fusions were identified and represented in Fig. 2. Five novel or rarely reported fusions were identified in six cases (Additional file 1: Table S7).

\section{Leukemia and lymphoma}

There were 134 panels performed on 124 different patients with leukemia and lymphoma. A total of 1147 variants (SNV/indels, CNVs, fusions) were classified into Tiers $1-4$, with $56.8 \%(651 / 1147)$ as Tier 1 and 2 variants. Overall, at least one Tier 1 or 2 variant was identified in $86.6 \%(116 / 134)$ of leukemias/lymphomas tested. Of those Tier 1 or 2 variants, CNVs were the most common alteration identified in $70.9 \%$ (95/134) of panels followed by SNVs/indels $(62.7 \%, 84 / 134)$ and fusions (23.9\%, 32/134). Among leukemias/lymphomas, gains and losses were distributed throughout the genome (Fig. 1). Clinically significant CNVs were more common in ALL, the most common of which was $-9 \mathrm{p}$ or loss of heterozygosity (LOH) of 9p including $C D K N 2 A, C D K N 2 B$, and $P A X 5$. Following were gains of chromosome 21 and $17 \mathrm{q}$ including $I K Z F 3$, $-7 \mathrm{p} / \mathrm{LOH}$ of $7 \mathrm{p}$ including IKZF1, -19p including TCF3, and -17p/LOH including TP53. The most commonly identified variants in leukemia/lymphoma specimens were in NRAS (12.7\%, 17/134), including patients with B-ALL [9], acute myelogenous leukemia (AML) [4], JMML [2], T-ALL [1], and T-myeloid leukemia [1] (Fig. 5). The majority of these patients also had variants and/or fusions identified in other genes tested. KRAS hotspot variants in codons 12, 13, and 61 were the second most common finding, present exclusively in patients with B-ALL, followed by variants in PTPN11, TP53, and NOTCH1 (Fig. 5). When classified by tumor type, the majority of variants in B-ALL were in KRAS, NOTCH1, PAX5, CREBBP, PTPN11, and JAK2. In AML, the most common mutations identified were in NRAS, RUNX1, and FLT3. ETV6-RUNX1 was the most common fusion identified in 6.7\% (9/134) panels from patients with B-ALL (Figs. 2 and 5). Finally, 15 patients (12.1\%) had clinically significant pharmacogenomic variants detected in TPMT and NUDT15, including 1 ALL case with homozygous pharmacogenetic variants in NUDT15 that prompted alterations in thiopurine dosing.

\section{Clinical impact of genomic findings}

Following case review of each panel tested and the overall clinical impact of comprehensive results for a given patient, $78.7 \%$ of all patients derived diagnostic, prognostic, and/or therapeutic benefit from comprehensive genomic testing (Fig. 6a, Table 1, Additional file 3: Table S8). Importantly, this clinical impact classification includes all cases in which somatic tumor testing was incorporated into a comprehensive analysis of all clinical and pathologic information and contributed to cancer care; in some cases, findings would have been recapitulated with malignancy-specific testing. In all tumor types, testing most often impacted

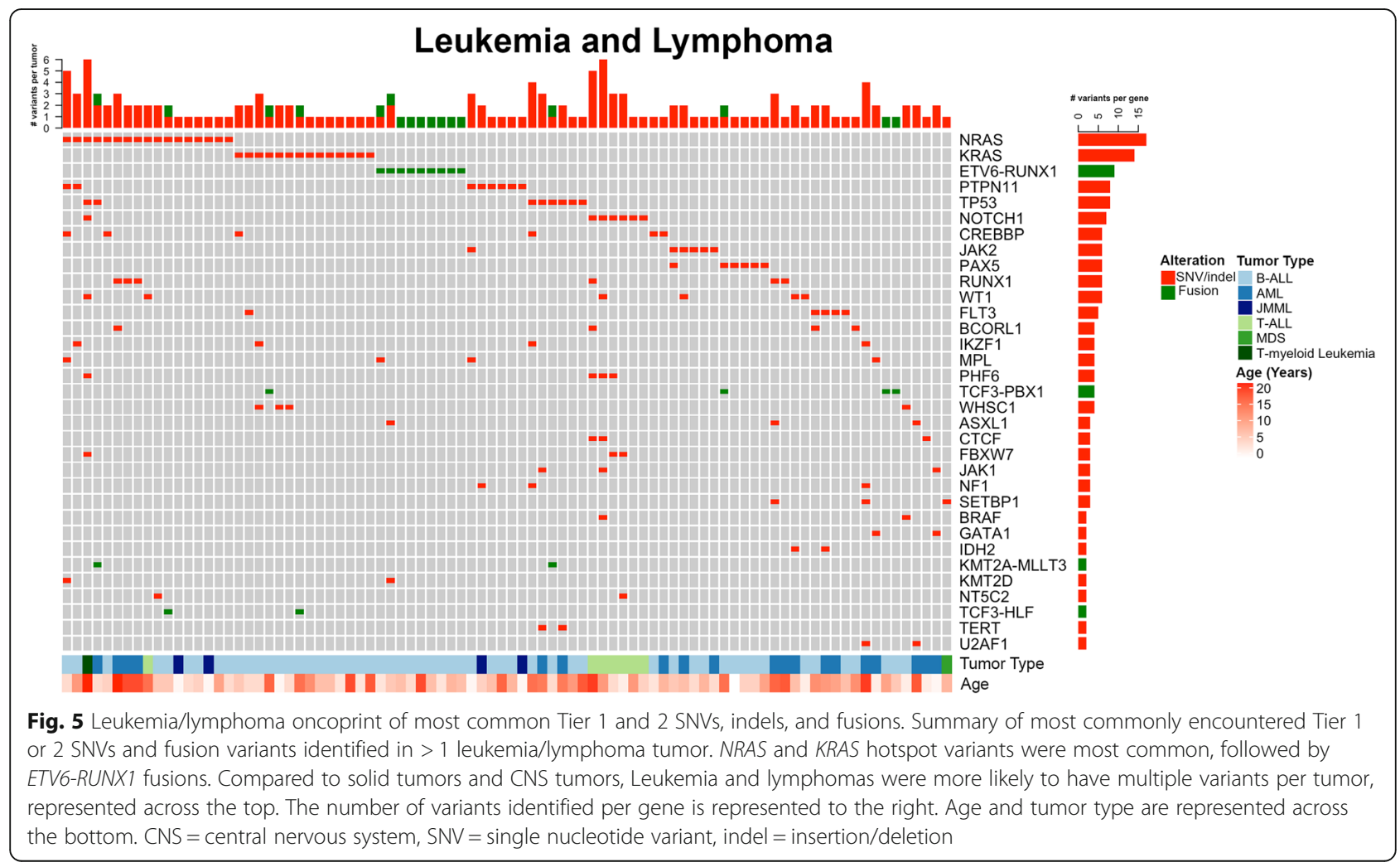




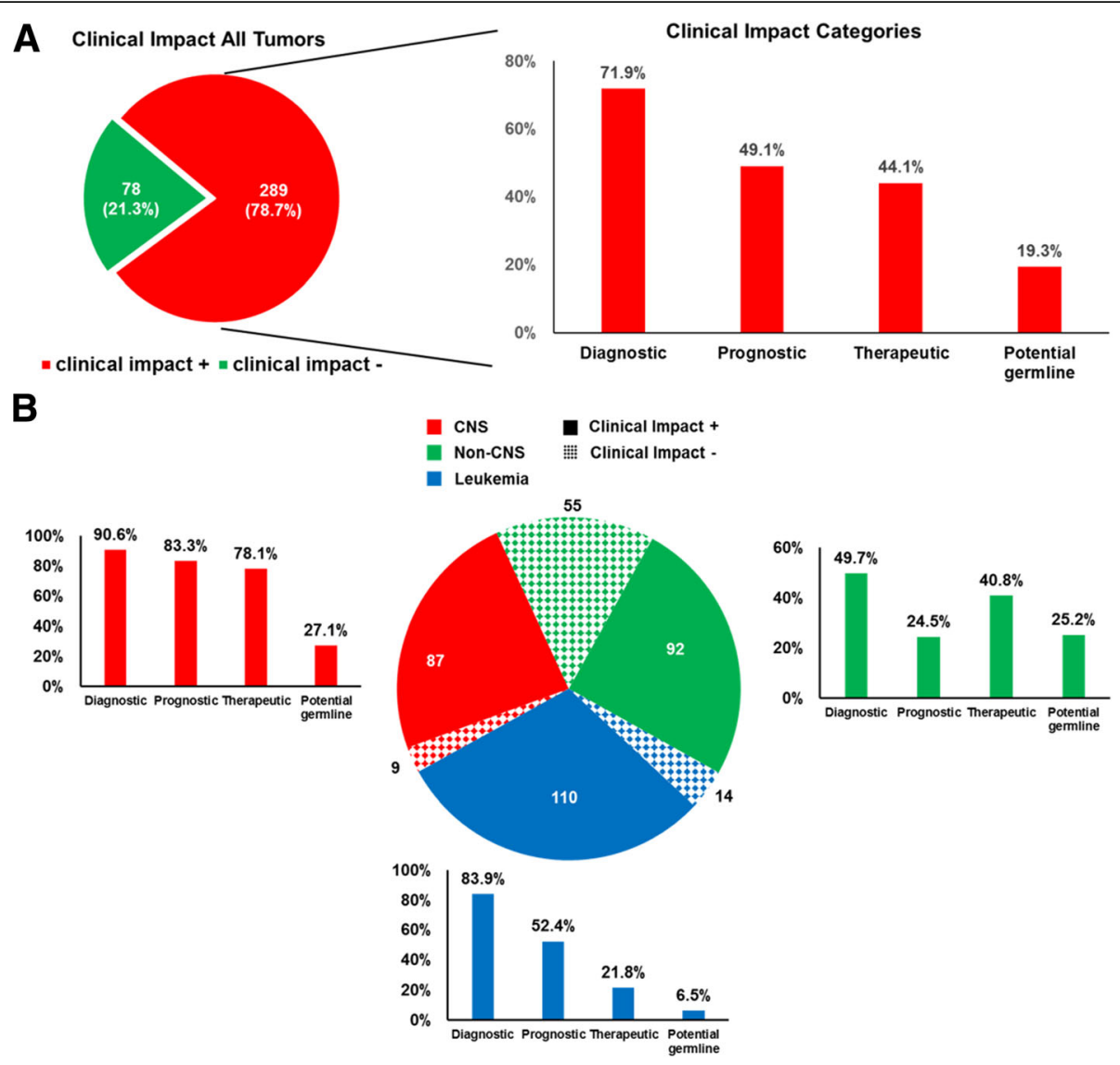

Fig. 6 Clinical impact of panel sequencing. Cases were considered positive for clinical impact (+) if they contained clinically significant Tier 1 or 2 variants. a. Overall, 289 cases (78.7\%) had positive clinical impact for at least one type of category (diagnostic, prognostic, therapeutic, potential germline). The bar chart on the right shows the percentage of cases with clinically significant Tier 1 or 2 variants impacting each category. $\mathbf{b}$. Summary of clinical impact color-coded by tumor type and impact category. Pie chart depicts number of patients in each slice. Hash marks indicate no clinical impact while solid color indicate positive clinical impact

patients by refining or changing the diagnosis (71.9\%). Genomic results were also particularly impactful for prognosis in $49.3 \%$ of patients. Therapeutic impact was examined in two ways: potential change to therapy (escalation, de-escalation, treatment stratification) based on prognostic genomic information and/or potential for targeted therapy based on genomic results. Genomic results had potential therapeutic impact by providing prognostic information in $35.7 \%$ of patients and potential targeted therapy options in $12.0 \%$ (Table 1). CNS tumors and leukemia/lymphomas were the two groups with the highest overall impact per patient (90.6\% and $88.7 \%$ overall, respectively), which is largely attributed to diagnostic and prognostic impact (Fig. $6 \mathrm{~b}$ and Table 1). Non-CNS solid tumors had a lower overall impact from genomic testing $(62.6 \%, p<0.001)$; however, they were more likely to have a potential therapeutic target identified $(21.8 \%, p<0.001)$ compared to CNS and leukemia/lymphoma groups $(5.2 \%$ and $5.7 \%$, respectively, Fig. $6 \mathrm{~b}$ and Table 1). Notably, this targeted option would be applicable in the relapse setting in most cases. A

Table 1 Clinical impact summary

\begin{tabular}{|c|c|c|c|c|c|c|c|c|}
\hline \multirow[t]{2}{*}{ Tumor type } & \multirow{2}{*}{$\begin{array}{l}\text { Number of } \\
\text { patients }\end{array}$} & \multirow{2}{*}{$\begin{array}{l}\text { Any clinical } \\
\text { Impact }\end{array}$} & \multicolumn{2}{|c|}{ Diagnostic impact } & \multirow{2}{*}{$\begin{array}{l}\text { Prognostic impact } \\
\text { Tier } 1 \text { and } 2\end{array}$} & \multicolumn{2}{|c|}{ Potential change to therapy } & \multirow{2}{*}{$\begin{array}{l}\text { Potential } \\
\text { Germline } \\
\text { alteration }\end{array}$} \\
\hline & & & Tier 1 and 2 & $\begin{array}{l}\text { Result changed } \\
\text { diagnosis }\end{array}$ & & $\begin{array}{l}\text { Change based } \\
\text { on prognosis }\end{array}$ & $\begin{array}{l}\text { Targeted } \\
\text { therapy }\end{array}$ & \\
\hline CNS & 96 & $87(90.6 \%)$ & $87(90.6 \%)$ & $3(3.1 \%)$ & $80(83.3 \%)$ & $75(78.1 \%)$ & $5(5.2 \%)$ & $26(27.1 \%)$ \\
\hline Leukemia/lymphoma & 124 & 110 (88.7\%) & $104(83.9 \%)$ & $4(3.2 \%)$ & $65(52.4 \%)$ & $22(17.7 \%)$ & $7(5.7 \%)$ & $8(6.5 \%)$ \\
\hline Solid (non-CNS) & 147 & $92(62.6 \%)$ & $73(49.7 \%)$ & $5(3.4 \%)$ & $36(24.5 \%)$ & $34(23.1 \%)$ & $32(21.8 \%)$ & $37(25.2 \%)$ \\
\hline Total & 367 & $289(78.7 \%)$ & $264(71.9 \%)$ & $12(3.3 \%)$ & $181(49.1 \%)$ & $131(35.7 \%)$ & $44(12.0 \%)$ & 71 19.3\%) \\
\hline
\end{tabular}


small but important finding is that genomic testing resulted in a diagnosis change across all tumor types in 12 patients (3.3\%, Additional file 3: Table S9). Finally, based on tumor-only sequencing, potential germline alterations were suspected in $19.4 \%$ of patients, all of whom were referred to the cancer predisposition program for genetic counseling and, in some cases, confirmatory germline testing. Of those that underwent confirmatory testing thus far, $53 \%$ had the somatic finding confirmed in the germline.

\section{Discussion}

We have developed and clinically validated large NGS-based panels for genomic profiling of pediatric tumors, similar, yet distinct, from other published panels for pediatric tumors [20,23, 38-40]. Our panels demonstrate high analytical validity with $>99.99 \%$ sensitivity, specificity, and reproducibility. The clinical utility and validity were evaluated using the 389 pediatric tumor samples tested during the first 15 months following implementation of these panels at our center. The spectrum of somatic variation observed and the suspected presence of germline predisposition were similar to that in larger cohort studies, with the caveat that not all suspected germline changes were confirmed to be germline on further analysis $[1,2,17,18,20-23,41]$. The number of clinically significant (Tier 1 or 2 ) sequence alterations per case is relatively low, especially in CNS and non-CNS solid tumors (Figs. 3, 4, and 5). This is a partial reflection of the low tumor mutational burden (TMB) observed in pediatric tumors in contrast to adult tumors, with the exception of pediatric samples containing pathologic variants in mismatch repair genes [3]. The general landscape of mutations observed from our cohort in large part matches data observed from recent large-scale panpediatric cancer studies [1, 2]. In particular, the most common significantly mutated genes that these studies identified (TP53, H3F3A, and CTNNB1) were also found to be highly recurrent in our patient cohort (Additional file 2: Figure S4, Figs. 3, 4, and 5).

Moving beyond overall trends in pediatric tumors, our results show that on a per-patient basis, comprehensive next-generation somatic tumor testing can be meaningfully incorporated into clinical care, as findings were clinically relevant in $78.7 \%$ of the patients tested in this cohort (Fig. 6 and Table 1). The rate of actionable alterations, including the rate of potentially germline alterations, is similar to that detected in other published clinical sequencing studies in pediatric oncology which show an approximate $30-60 \%$ rate of potentially targetable mutations, $\sim 10 \%$ rate of germline mutations with tumor/normal paired testing, and significant changes to diagnosis $[17,18,20-24,41]$. However, large-scale whole-exome sequencing and RNA-Seq is not currently clinically feasible on a large scale due to the cost and turnaround time. In addition, CSTP and CHTP use much less input DNA/RNA and provide much deeper sequence depth and higher sensitivity than that of whole-exome sequencing and RNA-Seq.

\section{Impact on diagnosis and prognosis}

Tumor genomic mutation profiles have started to play an important role in diagnosis, risk stratification, and prognostication in all pediatric cancers and in no area is that more significant than in CNS tumors [8]. Accordingly, panel testing was highly clinically relevant in CNS tumors, with $90.6 \%$ of results impacting diagnosis and $83.3 \%$ impacting prognosis, which is comparable to similar published studies and is largely due to the incorporation of molecular diagnostic criteria into the 2016 World Health Organization classification of CNS tumors $[8,40]$. Hematologic malignancies were the first adopters of genomic alterations in tumor diagnosis, classification, and risk stratification [42]. As such, panel testing in our cohort of leukemia/lymphoma patients had impact on diagnosis in $83.9 \%$ of cases, particularly in ALL and AML, and impacted prognosis in $52.4 \%$ of cases (Fig. $6 \mathrm{~b}$ and Table 1). In non-CNS solid tumors, $49.7 \%$ of somatic panels had diagnostic relevance, particularly in neuroblastoma, Ewing sarcoma, and fusion-positive rhabdomyosarcoma. Prognostic impact for non-CNS solid tumors was $24.5 \%$, which likely reflects the current solid tumor staging systems that are largely based on histologic tumor grading and extent of lymph node involvement. Nonetheless, all neuroblastomas in our cohort showed prognostic impact from testing because the comprehensive nature of the panel allowed detection of MYCN amplification, segmental chromosomal gains/losses, and ploidy estimates from copy number analysis, all of which are important for risk stratification based on current guidelines [6].

While most of the time comprehensive genomic results supported a pathologist's impression of a tumor, in a small but meaningful subset of cases (12 patients, 3.3\% of total) testing resulted in a change in histologic diagnosis (Table 1). These cases represent a sizable impact on patient care as changing of diagnosis inevitably leads to changes in prognosis and therapy $[24,43]$. One case is particularly notable because the diagnosis of atypical Ewing sarcoma was made prior to the implementation of the comprehensive solid tumor panel and was supported by EWSR1 rearrangement by break apart FISH. However, at the time of relapse, the tumor was tested using the CSTP, which revealed a rare EWSR1-CREB3L3 fusion, most diagnostic of sclerosing epithelioid fibrosarcoma [44]. This diagnosis ultimately led to a change of therapy for the patient. 


\section{Impact on therapy}

There are few FDA-approved targeted cancer therapies available to pediatric patients, yet there are several tumor types in which targeted therapy (FDA-approved and off-label) is accepted as best practice in the upfront or relapse setting. This includes the use of MEK inhibitors in relapsed CNS tumors with $B R A F$ fusion, use of crizotinib, or other ALK inhibitors in relapsed neuroblastoma with an $A L K$ alteration, and the use of imatinib and dasatinib in Ph (+) ALL $[11,12,45,46]$. The number of targetable variants was highest in the non-CNS solid tumor cohort $(21.8 \%)$, including $A L K$ variants in neuroblastoma, NTRK fusions in several different tumor types (including papillary thyroid cancer, mammary analogue secretory carcinoma, and myofibroblastic sarcoma), for whom targeted therapy with crizotinib or TRK inhibition (larotrectinib, entrectinib) was either recommended or available in the relapse setting [47-49]. There were also several KRAS and BRAF fusions and variants in non-CNS solid tumor cases for which targeted therapy could be significant in the relapsed setting. In leukemia subjects, Ph-like alterations were identified in several patients, leading to therapy with imatinib or ruxolitinib. FLT3 internal tandem duplication (ITD) was identified in one patient with AML which led to up-staging and change in treatment (addition of gilteritinib). In CNS tumors, the most common targetable lesions were $B R A F$ fusions (with KIAA1540 and other partners) that retained the active $B R A F$ kinase domain, amenable to MAPK targeting with MEK inhibitors [50].

While not all of the identified targetable lesions were treated as such given the availability of effective front-line therapy, there is an increase in the incorporation of targeted agents up front (including subgrouping based on these alterations in most recent Children's Oncology Group high-risk neuroblastoma and acute lymphoblastic leukemia protocols) [51, 52]. Finally, variant testing in TPMT and NUDT15 was included in leukemia/lymphoma cases, given that certain polymorphisms in these genes lead to altered metabolism of the therapeutic agents, thioguanine and mercaptopurine [26, 53]. Knowing the relevant TPMT and NUDT15 polymorphisms early in therapy allows appropriate chemotherapeutic dosing, prior to onset of toxicity, which were identified in $12 \%$ of leukemia cases in this cohort.

\section{Cancer predisposition implications}

Somatic sequencing can also identify potential underlying germline variants and subsequent cancer predisposition syndromes. This is particularly important given that family history and tumor type alone are not adequate to pick up all cases of hereditary cancer predisposition [54, 55]. As such, the criteria for suspicion of germline aberration (described in methods) were intentionally broad, to ensure all potential cases were referred for further consideration; not all somatic changes identified required germline testing after clinical review. Both CNS and non-CNS solid tumors had approximately $25-27 \%$ rate of possible germline alterations requiring additional follow-up with the cancer predisposition program. The most common somatic mutation concerning for cancer predisposition was TP53, associated with Li-Fraumeni syndrome, followed by NF1, NF2, BRCA2, WT1, RB1, APC, and PTEN. While some of these alterations are somatic changes only and tumor testing alone is not sufficient to diagnose germline conditions, the panel results allow for timely identification of patients to refer for genetic counseling and potential germline testing for a variety of cancer predisposition syndromes that may have important clinical implications for the patient and/or family members. Of particular interest are the high frequency of BRCA2 variants identified in our cohort, which are emerging therapeutic targets in some tumors other than breast and ovarian cancer [56]. Studies have suggested that germline confirmation of somatic BRCA2 variants are relevant to evaluation and care of pediatric patients and their families [57]. In the future, pairing somatic cancer gene panel sequencing with a matched normal tissue (blood or fibroblast) would both improve precision and variant calling and definitively identify pathogenic germline variants.

\section{Comprehensive assay design and reporting strategy}

As a whole, pediatric tumors span multiple categories; thus, broad sequencing panels were designed to reduce cost, simplify laboratory procedures, and maximize clinical utility. The open-ended nature of the anchored multiplex PCR fusion assay was critical in the identification of a number of novel and/or rarely reported fusions, especially in our population of rare tumor types. In some cases, while these fusions were novel, they were consistent with their suspected histologic diagnosis based on one or both fusion partners and predicted function of the fusion genes. For example, a novel MTP-BRAF fusion was consistent with the histologic diagnosis of Langerhan cell sarcoma, and TFG-ROS1 was consistent with an abdominal inflammatory myofibroblastic tumor. In other cases, novel/rare fusions allowed refinement or a change to diagnosis that would not have been possible using FISH or RT-PCR methodologies, such as the EWSR1-CREB3L3 fusion discussed above. In addition, the comprehensive assays were specifically designed to detect copy number variations as well as ITDs. Obtaining copy number information from NGS data is becoming increasingly common, with published methodologies from various groups [10, 58, 59]. Copy number alterations were the most commonly detected variant in our cohort and have important prognostic significance in tumors such as neuroblastoma and B-ALL, mirroring 
findings from other studies [9, 17]. Copy number analysis is also helpful when interpreting variant allele fraction in the context of tumor percentage. Additionally, we incorporated specific bioinformatics approaches to detect ITDs, especially those in FLT3 and BCOR. This integrated approach has allowed us to achieve efficient and comprehensive clinical impact from a variety of tumor types while simplifying lab procedures, reducing cost and TAT.

Our laboratory interpreted and reported sequence, fusion, and copy number variants in a single report using the published guidelines for sequence variant interpretation in cancer [36]. These results were reviewed by the treating oncologists and, when relevant, discussed at our institutional molecular tumor board. This rubric proved to be helpful to determine the clinical utility of test results due to the interpretation on a tumor-specific level. While few pediatric tumors have FDA-approved targeted therapy, many genomic variants have Tier 1 diagnostic and/or prognostic significance. Clinically, oncologists were able to use the tiered results for decision-making.

\section{Conclusions}

Use of somatic comprehensive panel testing had a meaningful impact on clinical care, including diagnosis, prognosis, and treatment planning in $78.7 \%$ of pediatric patients tested at our institution. Somatic NGS testing was efficiently incorporated into clinical care, providing comprehensive SNV/indel, copy number, and fusion information within a single report.

\section{Additional files}

Additional file 1: Table S1. Genes included in different panels. Table S2. Extraction methods used for the study. Table S3. Analytic performance on HapMap sample NA12878 for all known SNVs and indels in the ROI. Table S4. All known SNVs/indels and CNVs detected in the clinical samples used for validation. Table S5. Dilution studies for SNV/indel detection limit. Table S6. Reproducibility of the NGS Panels. Table S7. Novel/rarely reported fusions identified in 389 pediatric tumors. (DOCX $42 \mathrm{~kb}$ )

Additional file 2: Figure S1. Next-generation sequencing workflow. Figure S2. Average sequence depth of exons for genes in the hematologic and solid tumor panels. Figure S3. Dilution studies for copy number variant detection limit Figure S4. Frequency of Tier 1 or 2 variants detected in 101 genes across all tumor types. (PPTX $4638 \mathrm{~kb}$ )

Additional file 3: Table S8. Tier 1-3 variants detected in all cases with clinical utility scoring. Table $\mathbf{5 9}$. Twelve subjects that received a change to diagnosis as a result of genomic testing. (XLSX $352 \mathrm{~kb}$ )

\footnotetext{
Abbreviations

ALL: Acute lymphoblastic leukemia; AML: Acute myelogenous leukemia; CHMP: Comprehensive Hematological Malignancy Panel; CNS: Central nervous system; CNV: Copy number variation; CSTP: Comprehensive Solid Tumor Panel; FDA: Food and Drug Administration; FN: False negative; ITD: Internal tandem dubplication; indel: Small insertion/deletion; JMML: Juvenile myelomonocytic leukemia; LOH: Loss of heterozygosity; Ph (+): Philadelphia chromosome positive; ROI: Regions of interest; SNV: Single nucleotide variant; TP: True positive; TAT: Turn around time; VAF: Variant allele frequency; WHO: World Health Organization
}

\section{Acknowledgements}

The authors would like to thank Dr. Robert Doms MD PhD for his thoughtful review and feedback on this manuscript.

\section{Funding}

This work was supported by the Children's Hospital of Philadelphia.

\section{Availability of data and materials}

Most data (all variants identified as Tier 1, 2, and 3 and clinical variant annotation, including all data used to calculate clinical utility) generated or analyzed during this study are included in this published article and its supplementary information files. Full datasets generated and analyzed are not publically available due to potential compromise of individual privacy but are available from the author on reasonable request, including Tier 4 (benign/likely benign) variants. All code used to analyze datasets are available in a github repository (https://github.com/chopdgd/CHOP_CancerPanel_Analysis).

\section{Authors' contributions}

MML, FC, FL, RA, RB, JM, YM, MSan, SKT, AJW, JMM, and SPH all contributed to panel assay design and gene curation. KC and MSar developed the in-house bioinformatics pipeline. Clinical utility evaluation was performed collaboratively by LFS, SPM, GTA, GD, RB, MSan, SKT, and AJW. AG, MSar, PR, KSR, LFS, SPM, and MML were involved in data collection, curation, and analysis. LFS, SPM, and MML were major contributors in writing the manuscript. All authors read and approved the final manuscript.

\section{Ethics approval and consent to participate}

This study was approved by the Children's Hospital of Philadelphia Institutional Review Board as a retrospective analysis (IRB 17-013802). The IRB granted a waiver of consent and assent due to the retrospective nature of this study and no more than minimal risk (breach of confidentiality) to subjects. However, this waiver of consent or assent does not allow depositing full/raw datasets into publically available databases due to risk of breach of confidentiality (see below). This study was conducted in accordance with the Declaration of Helsinki.

\section{Consent for publication}

Not applicable.

\section{Competing interests}

The authors declare that they have no competing interests.

\section{Publisher's Note}

Springer Nature remains neutral with regard to jurisdictional claims in published maps and institutional affiliations.

\section{Author details}

${ }^{1}$ Department of Pathology and Laboratory Medicine, The Perelman School of Medicine at the University of Pennsylvania, Philadelphia, PA 19104, USA.

2Division of Genomic Diagnostics, Children's Hospital of Philadelphia, Philadelphia, PA 19104, USA. ${ }^{3}$ Division of Oncology, Children's Hospital of Philadelphia, Philadelphia, PA 19104, USA. ${ }^{4}$ Center for Data-Driven Discovery in Biomedicine, Children's Hospital of Philadelphia, Philadelphia, PA, USA. ${ }^{5}$ Center for Childhood Cancer Research, Children's Hospital of Philadelphia, Philadelphia, PA, USA. ${ }^{6}$ Department of Biomedical and Health Informatics, Children's Hospital of Philadelphia, Philadelphia, PA, USA. 'Department of Pediatrics, The Perelman School of Medicine at the University of Pennsylvania, Philadelphia, PA 19104, USA. ${ }^{8}$ Department of Pathology and Laboratory Medicine, The Children's Hospital of Philadelphia, The University of Pennsylvania Perelman School of Medicine, 3615 Civic Center Blvd., ARC 716i, Philadelphia, PA 19104, USA.

Received: 21 November 2018 Accepted: 7 May 2019

Published online: 28 May 2019

\section{References}

1. Grobner SN, Worst BC, Weischenfeldt J, Buchhalter I, Kleinheinz K, Rudneva VA, et al. The landscape of genomic alterations across childhood cancers. Nature. 2018;555:321-7.

2. Ma X, Liu Y, Liu Y, Alexandrov LB, Edmonson MN, Gawad C, et al. Pancancer genome and transcriptome analyses of 1,699 paediatric leukaemias and solid tumours. Nature. 2018;555:371-6. 
3. Alexandrov LB, Nik-Zainal S, Wedge DC, Aparicio SAJR, Behjati S, Biankin $A V$, et al. Signatures of mutational processes in human cancer. Nature. 2013;500:415-22.

4. Lawrence MS, Stojanov P, Polak P, Kryukov GV, Cibulskis K, Sivachenko A, et al. Mutational heterogeneity in cancer and the search for new cancerassociated genes. Nature. 2013:499:214-8.

5. Chalmers ZR, Connelly CF, Fabrizio D, Gay L, Ali SM, Ennis R, et al. Analysis of 100,000 human cancer genomes reveals the landscape of tumor mutational burden. Genome Med. 2017;9:34.

6. Cohn SL, Pearson AD, London WB, Monclair T, Ambros PF, Brodeur GM, et al. The International Neuroblastoma Risk Group (INRG) classification system: an INRG Task Force report. J Clin Oncol. 2009;27:289-97.

7. Pui CH, Carroll WL, Meshinchi S, Arceci RJ. Biology, risk stratification, and therapy of pediatric acute leukemias: an update. J Clin Oncol. 2011;29:551-65.

8. Louis DN, Perry A, Reifenberger G, von Deimling A, Figarella-Branger D, Cavenee WK, et al. The 2016 World Health Organization classification of tumors of the central nervous system: a summary. Acta Neuropathol. 2016; 131:803-20.

9. Sholl LM, Do K, Shivdasani P, Cerami E, Dubuc AM, Kuo FC, et al. Institutional implementation of clinical tumor profiling on an unselected cancer population. JCI Insight. 2016;1:e87062.

10. Cheng DT, Mitchell TN, Zehir A, Shah RH, Benayed R, Syed A, et al. Memorial Sloan Kettering-Integrated Mutation Profiling of Actionable Cancer Targets (MSK-IMPACT): a hybridization capture-based next-generation sequencing clinical assay for solid tumor molecular oncology. J Mol Diagn. 2015;17:251-64.

11. Bresler SC, Weiser DA, Huwe PJ, Park JH, Krytska K, Ryles H, et al. ALK mutations confer differential oncogenic activation and sensitivity to ALK inhibition therapy in neuroblastoma. Cancer Cell. 2014;26:682-94.

12. Maese L, Tasian SK, Raetz EA. How is the Ph-like signature being incorporated into ALL therapy? Best Pract Res Clin Haematol. 2017;30:222-8.

13. Mullard A. NCl-MATCH trial pushes cancer umbrella trial paradigm. Nat Rev Drug Discov. 2015;14:513-5.

14. FDA unveils a streamlined path for the authorization of tumor profiling tests alongside its latest product action. https://www.fda.gov/newsevents/ newsroom/pressannouncements/ucm585347.htm.: FDA; 2017.

15. CMS finalizes coverage of next generation sequencing tests, ensuring enhanced access for cancer patients. CMS.gov; 2018.

16. Joseph L, Cankovic M, Caughron S, Chandra P, Emmadi R, Hagenkord J, et al. The spectrum of clinical utilities in molecular pathology testing procedures for inherited conditions and cancer: a report of the Association for Molecular Pathology. J Mol Diagn. 2016;18:605-19.

17. Oberg JA, Glade Bender JL, Sulis ML, Pendrick D, Sireci AN, Hsiao SJ, et al. Implementation of next generation sequencing into pediatric hematology-oncology practice: moving beyond actionable alterations. Genome Med. 2016;8:133.

18. Serenius F, Ewald U, Faroogi A, Fellman V, Hafstrom M, Hellgren K, et al. Neurodevelopmental outcomes among extremely premature infants 6.5 years after active perinatal care in Sweden. JAMA Pediatr. 2016;170:954-63.

19. Parsons DW, Angshumoy R, Yang Y, Wang T, Scollon S, Bergstrom K, et al. Diagnostic yield of clinical tumor and germline whole-exome sequencing for children with solid tumors. JAMA Oncology 2016;epublished ahead of brint.

20. Pincez T, Clement N, Lapouble E, Pierron G, Kamal M, Bieche I, et al. Feasibility and clinical integration of molecular profiling for target identification in pediatric solid tumors. Pediatr Blood Cancer. 2017;64: e26365

21. Mody RJ, Wu YM, Lonigro RJ, Cao X, Roychowdhury S, Vats P, et al. Integrative clinical sequencing in the management of refractory or relapsed cancer in youth. JAMA. 2015;314:913-25.

22. Harttrampf AC, Lacroix L, Deloger M, Deschamps F, Puget S, Auger N, et al. Molecular Screening for Cancer Treatment Optimization (MOSCATO-01) in pediatric patients: a single-institutional prospective molecular stratification trial. Clin Cancer Res. 2017;23:6101-12

23. Harris MH, DuBois SG, Glade Bender JL, Kim A, Crompton BD, Parker E, et al. Multicenter feasibility study of tumor molecular profiling to inform therapeutic decisions in advanced pediatric solid tumors: the Individualized Cancer Therapy (iCat) study. JAMA Oncol. 2016:2(5):608-15.

24. Forrest SJ, Geoerger B, Janeway KA. Precision medicine in pediatric oncology. Curr Opin Pediatr. 2018;30:17-24.

25. Bell RJ, Rube HT, Xavier-Magalhaes A, Costa BM, Mancini A, Song JS, et al. Understanding TERT promoter mutations: a common path to immortality. Mol Cancer Res. 2016;14:315-23.
26. Relling MV, Hancock ML, Rivera GK, Sandlund JT, Ribeiro RC, Krynestski EY, et al. Mercaptopurine therapy intolerance and heterozygosity at the thiopurine Smethyltransferase gene locus. J Natl Cancer Inst. 1999;91:2001-9.

27. Yang JJ, Landier W, Yang W, Liu C, Hageman L, Cheng C, et al. Inherited NUDT15 variant is a genetic determinant of mercaptopurine intolerance in children with acute lymphoblastic leukemia. J Clin Oncol. 2015;33:1235-42.

28. Li H, Handsaker B, Wysoker A, Fennell T, Ruan J, Homer N, et al. The sequence alignment/map format and SAMtools. Bioinformatics. 2009;25:2078-9.

29. Garrison E, Marth G. Haplotype-based variant detection from short-read sequencing arXiv:12073907 [q-bioGN]; 2012.

30. Koboldt DC, Zhang Q, Larson DE, Shen D, McLellan MD, Lin L, et al. VarScan 2: somatic mutation and copy number alteration discovery in cancer by exome sequencing. Genome Res. 2012;22:568-76.

31. Cibulskis K, Lawrence MS, Carter SL, Sivachenko A, Jaffe D, Sougnez C, et al. Sensitive detection of somatic point mutations in impure and heterogeneous cancer samples. Nat Biotechnol. 2013;31:213-9.

32. Cingolani $P$, Platts $A$, Wang le $L$, Coon $M$, Nguyen $T$, Wang $L$, et al. A program for annotating and predicting the effects of single nucleotide polymorphisms, SnpEff: SNPs in the genome of Drosophila melanogaster strain W1118; iso-2; iso-3. Fly (Austin). 2012;6:80-92.

33. Cingolani P, Patel VM, Coon M, Nguyen T, Land SJ, Ruden DM, et al. Using Drosophila melanogaster as a model for genotoxic chemical mutational studies with a new program, SnpSift. Front Genet. 2012;3:35.

34. Stenson PD, Ball EV, Mort M, Phillips AD, Shiel JA, Thomas NS, et al. Human gene mutation database (HGMD): 2003 update. Hum Mutat. 2003;21:577-81.

35. Sherry ST, Ward MH, Kholodov M, Baker J, Phan L, Smigielski EM, et al. dbSNP: the NCBI database of genetic variation. Nucleic Acids Res. 2001 ; 29:308-11.

36. Li MM, Datto M, Duncavage EJ, Kulkarni S, Lindeman NI, Roy S, et al. Standards and guidelines for the interpretation and reporting of sequence variants in cancer: a joint consensus recommendation of the Association for Molecular Pathology, American Society of Clinical Oncology, and College of American Pathologists. J Mol Diagn. 2017;19:4-23.

37. Skinner ME, Uzilov AV, Stein LD, Mungall CJ, Holmes IH. JBrowse: a nextgeneration genome browser. Genome Res. 2009;19:1630-8.

38. Hiemenz MC, Ostrow DG, Busse TM, Buckley J, Maglinte DT, Bootwalla M, et al. OncoKids: a comprehensive next-generation sequencing panel for pediatric malignancies. J Mol Diagn. 2018;20:765-76.

39. Ishida H, Iguchi A, Aoe M, Takahashi T, Tamefusa K, Kanamitsu K, et al. Panel-based next-generation sequencing identifies prognostic and actionable genes in childhood acute lymphoblastic leukemia and is suitable for clinical sequencing. Ann Hematol. 2019;98:657-68.

40. Ramkissoon SH, Bandopadhayay P, Hwang J, Ramkissoon LA, Greenwald NF, Schumacher SE, et al. Clinical targeted exome-based sequencing in combination with genome-wide copy number profiling: precision medicine analysis of 203 pediatric brain tumors. Neuro-Oncology. 2017;19:986-96.

41. Parsons DW, Roy A, Yang Y, Wang T, Scollon S, Bergstrom $K$, et al. Diagnostic yield of clinical tumor and germline whole-exome sequencing for children with solid tumors. JAMA Oncol. 2016;2(5):616-24.

42. Sabittini E, Bacci F, Sagramoso C, Pileri S. WHO classification of tumours of the haematopoietic and lymphoid tissues in 2008: an overview. Pathologica. 2010;102:83-8.

43. Mody RJ, Prensner JR, Everett J, Parsons DW, Chinnaiyan AM. Precision medicine in pediatric oncology: lessons learned and next steps. Pediatr Blood Cancer. 2017;64.

44. Dewaele B, Libbrecht L, Levy G, Brichard B, Vanspauwen V, Sciot R, et al. A novel EWS-CREB3L3 gene fusion in a mesenteric sclerosing epithelioid fibrosarcoma. Genes, chromosomes \& cancer. 2017;56:695-9.

45. Kieran MW. Targeting BRAF in pediatric brain tumors. ASCO educational book; 2014. p. e438-3439.

46. Pikman $Y$, Stegmaier K. Targeted therapy for fusion-driven high-risk acute leukemia. Blood. 2018;132:1241-7.

47. Oral TRK inhibitor LOXO-101 (larotrectinib) for treatment of advanced pediatric solid or primary CNS tumors. clinicaltrials.gov: US National Library of Medicine $(\mathrm{NIH}) ; 2015$.

48. Study of RXDX-101 in children with recurrent or refractory solid tumors and primary CNS tumors with or without TRK, ROS1, or ALK fusions. clinicaltrials.gov: US National Library of Medicine (NIH); 2016.

49. Drilon A, Laetsch TW, Kummar S, DuBois SG, Lassen UN, Demetri GD, et al. Efficacy of larotrectinib in TRK fusion-positive cancers in adults and children. N Engl J Med. 2018;378:731-9. 
50. Banerjee A, Jakacki Rl, Onar-Thomas A, Wu S, Nicolaides T, Young Poussaint T, et al. A phase I trial of the MEK inhibitor selumetinib (AZD6244) in pediatric patients with recurrent or refractory low-grade glioma: a Pediatric Brain Tumor Consortium (PBTC) study. Neuro-Oncology. 2017:19:1135-44.

51. A phase III randomized trial for newly diagnosed high risk B-lymphoblastic leukemia including a stratum evaluating dasatinib (IND \#73789, NSC \#732517) in patients with Ph-like tyrosine kinase inhibitor (TKI) sensitive mutations. AALL1131. http://www.childrensoncologygroup.org/ The Children's Oncology Group; 2018.

52. A phase 3 study of 1311-Betaiodobenzyguanidine (1311-MIBG) or crizotinib added to intensive therapy for children with newly diagnosed high-risk neuroblastoma (NBL) IND\#134379. ANBL1531. http://www. childrensoncologygroup.org/ The Children's Oncology Group; 2018

53. Singh M, Bhatia P, Khera S, Trehan A. Emerging role of NUDT15 polymorphisms in 6-mercaptopurine metabolism and dose related toxicity in acute lymphoblastic leukaemia. Leuk Res. 2017;62:17-22.

54. Brodeur GM, Nichols KE, Plon SE, Schiffman JD, Malkin D. Pediatric cancer predisposition and surveillance: an overview, and a tribute to Alfred G. Knudson Jr. Clin Cancer Res. 2017;23:e1-5.

55. Zhang J, Walsh MF, Wu G, Edmonson MN, Gruber TA, Easton J, et al. Germline mutations in predisposition genes in pediatric cancer. N Engl J Med. 2015:373:2336-46.

56. Nieborowska-Skorska M, Sullivan K, Dasgupta Y, Podszywalow-Bartnicka P, Hoser G, Maifrede $S$, et al. Gene expression and mutation-guided synthetic lethality eradicates proliferating and quiescent leukemia cells. J Clin Invest. 2017:127:2392-406.

57. Walsh MF, Kennedy J, Harlan M, Kentsis A, Shukla N, Musinsky J, et al. Germline BRCA2 mutations detected in pediatric sequencing studies impact parents' evaluation and care. Cold Spring Harb Mol Case Stud. 2017;3: a001925.

58. Garcia EP, Minkovsky A, Jia Y, Ducar MD, Shivdasani P, Gong X, et al. Validation of Oncopanel: a targeted next-generation sequencing assay for the detection of somatic variants in cancer. Arch Pathol Lab Med. 2017;141:751-8

59. Shen W, Paxton CN, Szankasi P, Longhurst M, Schumacher JA, Frizzell KA, et al. Detection of genome-wide copy number variants in myeloid malignancies using next-generation sequencing. J Clin Pathol. 2018;71:372-8.

Ready to submit your research? Choose BMC and benefit from:

- fast, convenient online submission

- thorough peer review by experienced researchers in your field

- rapid publication on acceptance

- support for research data, including large and complex data types

- gold Open Access which fosters wider collaboration and increased citations

- maximum visibility for your research: over $100 \mathrm{M}$ website views per year

At $\mathrm{BMC}$, research is always in progress.

Learn more biomedcentral.com/submissions 